\title{
Actitud hacia la investigación y su importancia en la elección de la modalidad de tesis para optar el título profesional
}

\author{
Oscar Javier MAMANI BENITO'
}

\begin{abstract}
RESUMEN
El objetivo de esta revisión es destacar la importancia de una actitud favorable hacia la investigación en el proceso de elaboración de la tesis universitaria. La actitud es una predisposición para aceptar o no un objeto. Sus tres componentes como las creencias (cognitivo), sentimientos y emociones (afectivo) y la disposición conductual o comportamiento (conductual); permiten mediante sus propiedades como dirección e intensidad, mostrar si la actitud que se asume es positiva o negativa, tanto como favorable o desfavorable, en este caso hacia la investigación. En tal sentido la actitud hacia la investigación científica es una predisposición que se antepone al proceso de realización de una tesis; y dado que la conducta depende de las predisposiciones, una actitud positiva propiciará que los problemas se aborden sistemáticamente, que la información y las ideas se evalúen en forma crítica, haciéndose posible afrontar con eficacia el proceso de realizar una tesis de grado.
\end{abstract}

Palabras clave: Investigación científica, actitud, tesis.

\begin{abstract}
The aim of this review is to highlight the importance of a favorable attitude toward research in the process of elaboration of the university thesis. Attitude is a predisposition to accept or not an object. Its three components such as beliefs (cognitive), feelings and emotions (affective) and behavioral dispositions or behavior (behavioral), allowed through its properties as direction and intensity, show if the attitude is assumed to be positive or negative, as well as favorable or unfavorable, in this case to the investigation. In that sense the attitude toward scientific research is a predisposition that precedes the process of conducting a thesis, and since the behavior depends on the bias, conducive to a positive attitude that problems are addressed systematically, that the information and ideas critically evaluate it, becoming possible to effectively deal with the process of doing a thesis.
\end{abstract}

Keywords: Scientific research, attitude, thesis.

${ }^{1}$ Bachiller en Psicología, Estudiante del 3º de Teología con Mención en Psicología Pastoral, Universidad Peruana Unión. 


\section{INTRODUCCIÓN}

La educación superior es esencial para crear la capacidad intelectual de producir y utilizar conocimientos. En opinión de Cano (2002), uno de los retos más grandes en la vida académica de un estudiante es elaborar una tesis donde él o ella expresen sus ideas y hallazgos en el terreno profesional de su especialidad

Para Ramos y Sotomayor (2008), la tesis universitaria es un paso fundamental en la formación profesional y académica de un estudiante, ya que permite el desarrollo de nuevos conocimientos, facilita el dominio profesional y genera pensamiento crítico en los estudiantes al fomentar el aprendizaje basado en problemas. En el Perú, la tesis como actividad académica previa a la titulación en una carrera universitaria ha tenido diversas connotaciones con el transcurrir de los años; de ser un requisito para el bachillerato pasó a ser uno de los principales requisitos para la titulación, llegando en la actualidad a quedar como una de varias modalidades para obtener el título universitario.

La mayoría de universidades en el Perú permite al estudiante escoger entre dos modalidades para obtener un título: por un lado, el curso de actualización, que consta de una secuencia de sesiones de aprendizaje y evaluaciones escritas en áreas de especialización de la profesión y, por el otro, la tesis; siendo según San Martín y García (2006), el fundamento para este sistema el artículo $22^{\circ}$ de la ley universitaria $\mathrm{N}^{\circ} 23733$ (1983).

En opinión de Ángel (et al, 2010), la investigación estudiantil está enfrentando grandes dificultades, no solo en Latinoamérica sino en la mayoría de países del mundo. Si bien es cierto que se imparten cursos básicos de metodología de la investigación, se percibe que solo hay un grupo minoritario de alumnos que desean profundizar sus conocimientos y aplicarlos en la realización de trabajos de investigación, como es el caso de la tesis universitaria.

Desafortunadamente la producción de tesis en nuestro país es muy baja y aun más baja su publicación (Galan et al, 2005). Según un estudio realizado por San Martín y García (2006, citado por Valle y Salvador, 2009), a bachilleres recientemente graduados de una de las más prestigiosas universidades del Perú, como es la Universidad Nacional Mayor de San Marcos se observó que solo el $5 \%$ de todos los graduandos obtuvo el grado por un trabajo de tesis.

Siguiendo la misma línea de investigación en otras instituciones, una encuesta realizada por Frishman (2001), a un grupo de alumnos, dio a conocer que la mitad de los respondientes contestaron que la realiza- ción de una tesis no debería ser obligatoria, mientras que el $18 \%$ mencionó que sí debería serlo, y $33 \%$ tuvo una respuesta incierta en torno a la pregunta.

Finalmente en opinión de Guijon y Rodríguez (2000), la generación de nuevo conocimiento está condicionada por la preparación de las nuevas generaciones que se instruyen en el método científico, es en ese sentido que mediante este trabajo de revisión se pretende destacar la importancia de la actitud hacia la investigación por parte de los estudiantes universitarios, en la realización de sus tesis.

\section{Investigación}

Investigar proviene del latín "vestigio", literalmente "planta de pie" y por extensión la "huella" que queda. Sus sinónimos tales como indagar, inquirir, encaminar, inspeccionar, explorar, rastrear, buscar, coinciden en parte con el sentido de la palabra inglesa research.

\section{Investigación científica}

En opinión de Valverde (2005), es toda actividad humana orientada a descubrir algo desconocido; es el cuestionamiento del marco conceptual vigente que está relacionado con la búsqueda de soluciones de una necesidad manifiesta del hombre frente a sus problemas reales y potenciales. Según Hernández, Fernández y Baptista (2006), utilizando el método científico, dado por una serie de etapas sucesivas a seguir, se pretende alcanzar respuestas a interrogantes en diversas áreas de estudio como: la biológica, psicológica, social, económica o cognoscitiva, desde un contexto micro y macrocósmico.

En consecuencia, la investigación científica en el más amplio sentido de la palabra se define como la aplicación del método científico en la solución y la búsqueda de respuestas a interrogantes que se suscitan en la práctica profesional cotidiana; con ella se intenta resolver problemas en el sentido de explicar la relación de factores o acontecimientos.

La investigación estudiantil también es un proceso reflexivo, sistemático, veraz, crítico, de descubrimiento de verdades objetivas, de nuevos hechos, relaciones, dependencias con las leyes, principios generales y conocimientos de los fenómenos u objetos que se estudian (Hernández et al).

\section{Importancia de la investigación científica}

El ejercicio de la investigación en el proceso de generación de nuevos conocimientos es un factor totalmente decisivo de producción, riqueza y bienestar. Una de las razones sociales es el impacto en la educación, pues el nivel económico de los pueblos está fuertemente correlacionado con su educación. 
En nuestro país investigar es y debe de ser una aspecto muy importante de la capacidad para generar ciencia como paso previo a una sofisticada y fuerte organización tanto económica, como social. (Wasserman 2000, citado por Cotacallapa, 2010).

Para poder generar conocimiento nuestra mente debe estar debidamente entrenada y esto solo se puede lograr aprendiendo a investigar con una educación basada sobre la investigación científica que más que dar conocimientos, nos proporcione instrumentos analíticos y capacidad para producir hipótesis explicativas $\mathrm{y}$ funcionales que puedan dar origen a una sociedad que inventa.

\section{Tesis}

La palabra "tesis" proviene del latín "thesis" que significa: "Conclusión que se mantiene por razonamiento". Su uso más difundido corresponde al nivel de estudios superiores, donde constituye un trabajo académico, resultado de un proceso de formación ligado necesariamente a la investigación y presentada para obtener el título de licenciatura, maestría o doctorado.

En opinión de Parent (et al, 1995), una tesis es un postulado que se sostiene como verdad científica y que en las "Tesis de Graduación" va precedida de una descripción de todo el proceso de investigación que prueba su verdad. Ese postulado científico, a su vez, es la respuesta clara y resumida a aquello que fue planteado como Problema de Investigación.

Durante el siglo XX, principalmente en el mundo iberoamericano, por extensión se le ha dado el nombre de "tesis" a todo el documento que acompaña a la (o las) tesis postuladas, pero eso no es exacto. Este cambio de significado produce algunos errores garrafales, en el sentido de que muchos tesistas producen un gran volumen de investigación, pero al final de su trabajo no presentan ninguna "tesis" en la forma de postulado que resuma la respuesta al problema, porque desconocen qué es una tesis (Oficina de Grado y Títulos USMP, 2010).

\section{Situación de la tesis en el Perú}

En 1991 se expide el decreto legislativo $N^{0} 739$ que permite obtener el grado de bachiller en forma automática luego de haber aprobado el programa de estudio correspondiente (bachillerato correspondiente), a la vez que faculta la obtención del título universitario mediante tres modalidades:

1. Con la aprobación y presentación de una tesis.

2. Experiencia de tres años de trabajo posteriores al término del programa de estudio de la especialidad y la presentación de una monografía.
3. Cualquier otra modalidad que estime conveniente la universidad.

Como ejemplo de esta última modalidad, la titulación extraordinaria ha sido ampliamente utilizada, la cual consiste en atender y aprobar un modulo de cursos de corta duración. Aunque la mayoría de los programas académicos de licenciatura incluyen como requisito de titulación la elaboración de tesis de investigación, las opciones alternativas, que se presentan y que suelen ser múltiples, originan que la investigación científica a nivel de licenciatura sea prácticamente nula. Las tesis de investigación científica que se generan, y se publican generalmente provienen principalmente, de los programas de posgrado.

Sobre este caso, San Martín y García (2006) mencionan que la apertura de otras formas de titulación, diferentes a la presentación de una tesis, dio lugar en la práctica a la desaparición paulatina de la titulación bajo la modalidad de tesis en la mayoría de universidades del Perú, por no decir casi todas.

\section{Importancia de la Tesis Universitaria}

Durante mucho tiempo el realizar un buen trabajo de tesis constituía quizás lo principal en una formación universitaria. Para Soto (2005), la tesis representaba el momento culminante de la carrera, en donde el alumno demostraba de lo que era capaz; la solemnidad que rodeaba el acto de defender una tesis mostraba la importancia de ese momento, pues era escenario del nacimiento de un nuevo profesional.

En opinión de Ruiz (2005), la tesis universitaria también es una de las principales experiencias de aprendizaje del alumno y al mismo tiempo, representa un mecanismo adecuado para que las universidades certifiquen las competencias de sus graduados. Al respecto otra vez Soto (2005), resalta la tesis como una fuente de desarrollo de capacidades del individuo, pues en el trabajo de investigación el tesista puede descubrir habilidades, destrezas, aptitudes que antes desconocía, o bien, desarrollarlas si ya las tenía.

Además de infundir disciplina, plantearse metas y no descansar hasta alcanzarlas, se conlleva a la responsabilidad, a la capacidad de fórmularse bien los problemas, de saber recolectar datos pertinentes, de analizarlos y de diseñar soluciones; en consecuencia, muchas de las competencias que buscan los empresarios en los nuevos profesionales, se pueden desarrollar en el proceso de elaborar una tesis.

\section{Actitud}

Según el diccionario de la lengua española, de la Real Academia, en su $22^{\circ}$ edición (2001), la palabra "acti- 
tud" proviene del latín actitudo, que significa postura del cuerpo humano.

Para Eysenc (1980), las actitudes son una disposición evaluativo relativamente duradera hacia un objeto de vida, relativamente constante con respecto a cierta cosa ya sea neutral, desfavorable o favorable.

En opinión de Gibson, Ivancevich y Donelly (1981, citados por Chiavenato, 2004), refieren que la actitud es un estado mental que es organizado por la experiencia e influye de manera específica en la respuesta de la persona ante objetos, situaciones y otras personas. Finalmente, Morris (1992), menciona que las actitudes son el conjunto de disposiciones hacia el medio exterior, las formamos desde niños de acuerdo con las experiencias vividas con nuestros padres o seres más allegados.

Es cierto que se evidencia diversas definiciones realizadas por distintos teóricos, pero es necesario resaltar que "existe un consenso entre los teóricos en afirmar que la actitud: es una predisposición psicológica de comportarse de manera favorable o desfavorable frente a una entidad particular u objeto" (Cotacallapa, 2010 pág. 23).

En ese sentido, la actitud es una disposición fundamental que interviene e influye en la determinación de una diversidad de conductas hacia un objeto; siendo ésta fundamentalmente una disposición y participación compleja a responder favorable, medianamente favorable o desfavorablemente, adoptando una respuesta de aceptación o rechazo a determinadas situaciones, personas o cosas.

\section{Componentes}

Las actitudes poseen tres componentes básicos: "uno afectivo, otro cognoscitivo y un último conductual" (Gagne, 1987; Reich y Adcock, 1980; Sarabia, 1992; Javiedes, 1996; Morales, 2000, citados por Cotacallapa, 2010, pag 44).

1. Componente Cognitivo: es el más importante, pues se asegura que sin conocimiento no existe actitud. Todas las creencias acerca de un objeto se incluyen en el componente cognitivo, el número de elementos de este componente varía de una persona a otra, pues un individuo puede creer que muchas cosas acerca de un objeto son verdaderas; pero las creencias evaluativas son más importantes para la actitud como concepto de disposición, pues este último abarcan las creencias acerca de las cualidades deseables o indeseables, aceptables o inaceptables, o sencillamente buenas o malas.
2. Componente Emocional: muy conocido como el componente sentimental, se refiere a las emociones o sentimientos ligados con el objeto de la actitud. Los objetivos bipolares usados frecuentemente al estudiar los elementos de este componente son amor-odio, gusto-disgusto, admiracióndesprecio y otros que denoten un sentimiento de tipo favorable o desfavorable.

3. Componente Conductual: En éste se incorpora la disposición conductual del individuo a responder al objeto, ya que se acepta generalmente que hay un lazo entre los componentes cognitivos, particularmente las creencias que manifiestan una cualidad deseable o indeseable, aceptable o inaceptable, y éstas generan la disposición a responder al objeto, específicamente guían nuestro comportamiento.

\section{Propiedades}

Según Calenzani (1983; citado por Valverde, 2005), la tendencia de las actitudes está dada porque éstas pueden ser descritas de manera completa por medio de dos propiedades: La dirección de la actitud (positiva o negativa) y el grado del sentimiento representado (la intensidad):

a. Dirección: Las actitudes pueden estar orientadas positiva o negativamente con respecto a un objeto. Se entiende por "positiva" a la tendencia de acercamiento hacia el objeto de actitud, mientras que la "negativa" se refiere a la predisposición a evitar el mismo.

b. Intensidad: Está relacionada con el aspecto emocional de las actitudes lo cual determina los grados de intensidad con que se reacciona frente al objeto de la actitud, es decir el grado de sentimiento representado (favorable, medianamente favorable o desfavorable).

\section{Clasificación}

Una actitud puede ser clasificada, medida o valorada en:

- Favorable: consiste en estar de acuerdo con lo que se realiza, es decir el sujeto muestra cierta tendencia de acercamiento hacia el objeto, generalmente está unido con el sentimiento de apoyo, estimulación, ayuda y comprensión.

- Desfavorable: es cuando el sujeto evita el objeto, como motivo de la actitud, se presenta el recelo, la desconfianza y en algunos casos la agresión y frustración que generalmente lleva a la persona a un estado de tensión constante. 
- Medianamente favorable: es un sentimiento de apatía, el sujeto no muestra aceptación ni rechazo al objeto, prevalece el desinterés, la rutina y en algunos casos el aburrimiento.

\section{Actitudes hacia la investigación}

Valverde, (2005) la actitud hacia la investigación es la predisposición del estudiante universitario para actuar y/o participar en la investigación científica, mediante su capacidad y/o características inherentes, que posee para su desarrollo.

Dado que la conducta depende de las predisposiciones, una actitud positiva hacia la investigación propiciará que los problemas se aborden sistemáticamente y que la información y las ideas se evalúen en forma crítica, lo que en consecuencia dará a los alumnos mayores oportunidades de alcanzar mejores soluciones. Otra implicación importante para la adquisición de estas actitudes por parte de los estudiantes se refiere a su desarrollo personal, pues según Nobigrot, Kleinman y Col (1995), el estudiante llega a mostrar más interés en la búsqueda sistemática y organizada de soluciones a problemas cotidianos que se le presentan mayor tolerancia hacia otros puntos de vista y menor tendencia hacia la aceptación de conclusiones erróneas, al transferir esas actitudes a situaciones de la vida diaria

\section{CONCLUSIONES}

El paso por la universidad debe significar mucho más que solamente obtener un titulo profesional. La esencia del esfuerzo académico debe contribuir al cumplimiento de la función de la misma: "la producción de conocimiento nuevo"; y una de las mejores oportunidades que el estudiante tiene para demostrarlo; es mediante la realización de una investigación que conlleve a su tesis, como producto de su creatividad y competencia intelectual.
En la educación superior el énfasis en las actitudes positivas y favorables hacia la investigación ha sido enfocada, por diversos autores y producto de ello nace la importancia de prestar atención a las predisposiciones como factores que influyen en el estudiante, al momento de lograr o no una mejor comprensión de la naturaleza del proceso científico. Es en ese sentido que el valor de una actitud favorable hacia la investigación como proceso y como producto, permite al estudiante familiarizarse y alcanzar cierto dominio que le permita enfrentar y desarrollar con seguridad retos y requisitos académicos, siendo uno de ellos el optar la modalidad por la cual va a obtener el título universitario.

En ese sentido, la tesis representa el pasaje del estudiante al profesional, pues en este trabajo deberá probar a la universidad y a la sociedad que está preparado para asumir los desafíos del competitivo mundo laboral. Pero la tesis es también un aprendizaje para la vida. Un problema de investigación encierra la misma lógica de un problema personal: el individuo que es capaz de enfrentar exitosamente un problema de investigación profesional, puede hacer lo mismo con un problema personal.

Se hace necesario enfatizar las predisposiciones con las que el estudiante cuenta al momento de realizar o no una tesis, ya que una actitud positiva propiciará que los problemas se aborden sistemáticamente, que la información y las ideas se evalúen en forma crítica; haciéndose posible afrontar con eficacia el proceso de realizar una investigación científica que conlleve a defender y sustentar una tesis.

Finalmente, la promoción de predisposiciones positivas hacia la investigación debe ser parte de objetivos implícitos o explícitos en diversos los proyectos curriculares. Especialmente la evaluación de las actitudes deseables puede conducir al diagnóstico que fundamente su aceptación o, en caso contrario, que permita proponer estrategias para remediar las actitudes no deseadas. 


\section{REFERENCIAS BIBLIOGRÁFICAS}

1. Angel, A; Botero, H; Gonzales, D; Piedad, L; Velasco, M; y Ocampo, M. (2010). Interés de los Estudiantes de Medicina por la Investigación. CIMEL, Vol. 15, Nº 1, 9-13

2. Cano, Santana, Zenon. (2002). ¿Cómo escribir una tesis? CIENCIA-UNAM pag 68-75

3. Cotacallapa, D. (2010). Estilos de aprendizaje y actitudes hacia la investigación en los estudiantes de enfermería de la Universidad Nacional Hermilio Valdizan de Huanuco. Tesis inédita de Posgrado en Educación, Universidad Peruana Unión, Perú.

4. Chiavenato, Idalberto. (2004). Comportamiento Organizacional. Mexico: Editorial Thomson.

5. Direccion de la Oficina de Grados y Titulos. (2010), Elaboracion, Estructura y Presentacion de la Tesis Universitaria. Universidad San Martín de Porres, Recuperado de: http://www. usmp.edu.pe/odonto/MANUAL.pdf

6. Eysenc, G. (1980). Texto de Psicología Humana. Mexico / Argentina: Editorial Manual Moderno.

7. Frishman W. H. (2001) Student research projects and theses. should they be a requirement for medical school graduation? Heart Dis; 3(3): 14044.

8. Galán, E; Manrique, N; Villavicencio, E; Yllatopa, E; Peralta, M; De la Cruz, W. (2005). Producción cientifica de los investigadores del pregrado de Medicina Humana del Perú, 19932003. CIMEL v.10 n.1.

9. Morris, G. (1992). Psicología un nuevo enfoque. México: Prentice Hall

10. Nobigrot, Kleinman y Col (1995). Las actitudes hacia la investigación y el aprendizaje en estudiantes de medicina. Salud Pública de México. Recuperado de: http://redalyc.
uaemex.mx/redalyc/src/inicio/ArtPdfRed. jsp? iCve $=10637406$

11. Parent, J; Farrand, J; Esquivel, N; Peña, R; y Pérez, E. (1995). ¿Qué es la tesis?. Centro de estudios UNAM. Mexico:Toluca

12. Ramos-Rodríguez, Mariana; Sotomayor, Renzo Realizar o no una tesis: razones de estudiantes de medicina de una universidad pública y factores asociados. Rev. perú. med. exp. salud pública v. 25 n.3

13. Rodríguez-Paz, C.A; y Gijón Granados, E. (2000) Investigacion durante la licenciatura. Rev Fac Med UNAM Vol. 43, No 3, 87-89

14. Ruiz Dodobara, Fernando (2005). Relación entre la motivación de logro académico, la autoeficacia y la disposición para la realización de una tesis. Persona, Núm. 8, pp. 145-170

15. San Martín, F; García P., M. (2006) La Tesis y su problemática en la Facultad de Medicina Veterinaria de la UNMSM. Rev Inv Vet Perú 2006; 17 (1): 81-88

16. Soto Acosta, W. (2005). Muéstreme su tesis y le diré de qué es capaz. Revista Comunicación. Vol 14, No 2, 69-73

17. Valverde, M. (2005). Actitud de las enfermeras hacia la investigación y factores que intervienen en su realización en el Hospital Nacional Daniel Alcides Carrión. Tesis Inédita de pregrado, UNMSM, Perú.

18. Valle, Rubén; Salvador, Elisa (2009). La Tesis de Medicina Humana: Experiencia en una Universidad Pública de Lima. Rev Peru Med Exp Salud Publica; 26(2): 268-70. Angel, A; Botero, H; Gonzales, D; Piedad, L; Velasco, M; y Ocampo, M. (2010). Interés de los estudiantes de Medicina por la investigación. CIMEL, Vol. $15, \mathrm{~N}^{\mathrm{o}} 1,9-13$ 